\title{
Survival benefit of chemoembolization plus Iodine125 seed implantation in unresectable hepatitis B-related hepatocellular carcinoma with PVTT: a retrospective matched cohort study
}

\author{
Mingsheng Huang ${ }^{1,2,3} \cdot$ Qu Lin ${ }^{4}$ Haofan Wang ${ }^{1,2,3} \cdot$ Junwei Chen ${ }^{1,2,3} \cdot$ \\ Mingjun Bai ${ }^{1,2,3} \cdot$ Long Wang ${ }^{1,2,3} \cdot$ Kangshun Zhu $^{1,2,3}$ - Zaibo Jiang ${ }^{1,2,3}$. \\ Shouhai Guan ${ }^{1,2,3} \cdot$ Zhengran $\mathrm{Li}^{1,2,3} \cdot$ Jiesheng Qian ${ }^{1,2,3} \cdot$ Mingan $\mathrm{Li}^{1,2,3}$. \\ Pengfei Pang ${ }^{1,2,3} \cdot$ Hong Shan ${ }^{1,2,3}$
}

Received: 31 May 2015 / Revised: 22 December 2015 / Accepted: 30 December 2015 / Published online: 20 January 2016 (C) The Author(s) 2016. This article is published with open access at Springerlink.com

\begin{abstract}
Objectives To investigate the survival benefit of transarterial chemoembolization (TACE) plus Iodine125 seed implantation (TACE-Iodine125) in hepatitis B-related HCC patients with portal vein tumour thrombus (PVTT) and the underlying prognostic factors.

Methods A retrospective matched cohort study was performed on consecutive HCC patients with PVTT from January 2011 to June 2014. Seventy patients (TACEIodine125 group) who underwent TACE-Iodine125 were compared with a historical case-matched control group of 140 patients (TACE group) who received TACE alone. The survival of patients and the underlying prognostic factors were analysed.

Results The median survival times of the TACE-Iodine125 and TACE groups were 11.0 and 7.5 months, respectively $(p<0.001)$. The survival probability at 12,24 , and 36 months
\end{abstract}

Mingsheng Huang, Qu Lin and Haofan Wang contributed equally to this work.

Mingsheng Huang

huangmsh@mail.sysu.edu.cn

Hong Shan

shanhong@mail.sysu.edu.cn

1 Department of Radiology, the Third Affiliated Hospital, Sun Yat-sen University, 600 Tianhe Road, Guangzhou 510630, China

2 Department of Interventional Radiology, Ling-nan Hospital, Sun Yat-Sen University, Guangzhou, China

3 Interventional Radiology Institute, Sun Yat-sen University, Guangzhou, China

4 Department of Oncology, the Third Affiliated Hospital, Sun Yat-sen University, Guangzhou, China was $50 \%, 14.5 \%$, and $14.5 \%$ vs. $25 \%, 9 \%$, and $5 \%$ in the TACE-Iodine125 and TACE groups, respectively $(p<0.001)$. The PVTT responders had better survival than the PVTT nonresponders $(p<0.001)$. For the PVTT non-responders, there were no differences in the survival curves between the groups $(p=0.353)$. Multivariate analysis showed that type III PVTT $(p<0.001)$ and APS $(p<0.001)$ were independent predictors of poor prognosis. In contrast, the treatment modality of TACE-Iodine125 $(p<0.001)$ and PVTT response $(p=0.001)$ were favourable prognostic features.

Conclusions TACE combined with Iodine125 seed implantation may be a good choice for selected HB-HCC patients with PVTT.

Key Points

- TACE-Iodine125 was more effective than TACE for patients with HCC-PVTT.

- The TACE-Iodine125 procedure was safe.

- TACE-Iodine125 was conditional for patients with HCCPVTT.

- TACE-Iodine125 resulted in a better PVTT response compared to TACE alone.

- A good PVTT response is a favourable prognostic factor.

Keywords Hepatocellular carcinoma · Portal vein tumour thrombus $\cdot$ Interventional therapy $\cdot$ Brachytherapy $\cdot$ Survival

$\begin{array}{ll}\text { Abbreviations } \\ \text { TACE } & \text { Transarterial chemoembolization } \\ \text { PVTT } & \text { Portal vein tumour thrombus } \\ \text { HB-HCC } & \text { Hepatitis B-related hepatocellular carcinoma } \\ \text { OS } & \text { Overall survival } \\ \text { APS } & \text { Arterial-portal-shunt }\end{array}$




$\begin{array}{ll}\text { HCC } & \text { Hepatocellular carcinoma } \\ \text { BCLC } & \text { Barcelona clinic liver cancer } \\ \text { ECOG } & \text { Eastern cooperative group performance status } \\ \text { AFP } & \text { Alpha fetoprotein } \\ \text { ALT } & \text { Alanine transaminase } \\ \text { GGT } & \text { Glutamyltransferase } \\ \text { TBIL } & \text { Total bilirubin }\end{array}$

\section{Introduction}

Hepatocellular carcinoma (HCC) is the sixth most common malignancy and the third leading cause of death of all malignancies worldwide [1]. The majority of HCC in southern China is related to hepatitis B (HB) [2]. Portal vein tumour thrombus (PVTT) is a common complication of HCC that occurs in approximately $10-40 \%$ of HCC patients at the time of diagnosis [3]. PVTT may cause diffuse intrahepatic metastases and aggravate liver damage. PVTT has been proposed to be the primal and independent predictor of poor prognosis in HCC patients [4]. If untreated, HCC patients with PVTT (HCC-PVTT) have a median overall survival (OS) of only 2.7-4 months, whereas patients without PVTT have a median OS of 10-24 months [3].

Molecular targeted agents such as sorafenib were recommended as the standard treatment for HCC and PVTT patients $[5,6]$, and were shown to increase the median OS to 6.5 months in a phase III, randomized controlled trial in Asia [7]. Regular application of sorafenib is limited in China because many patients cannot afford the expensive cost. Additionally, sorafenib is poorly tolerated in some patients, as reflected by a discontinuation rate of 15-38 \%, owing to adverse events [7, 8]. In Asia, transarterial chemoembolization (TACE) is still considered a useful treatment for patients with unresectable HCC and PVTT [9]. Because the tumour-feeding PVTT vessels mostly originate from the hepatic artery, TACE for PVTT has proven to be effective, with a median survival time of 7.1-9.5 months [10,11]. However, the impact of TACE alone on PVTT is limited because it is hard to completely embolize the PVTT feeding vessels, and the response to TACE is poor when PVTT is complicated with arterial-portal-shunt (APS)[11, 12].

Studies have shown that HCC is radiosensitive [13]. Iodine 125 seeds are an internal radiation source that have been widely applied for the treatment of prostate cancer,head and neck tumours and HCC $[14,15]$. Iodine 125 brachytherapy alone showed promising efficacy for unresectable HCC with PVTT [14]. We hypothesize that TACE and Iodine125 seed implantation have synergistic effects on HCC with PVTT. However, few reports have focused on the effects of combined TACE and Iodine125 seed implantation (TACE-Iodine125) for HCC with PVTT. In this study, we conducted a retrospective matched cohort study to evaluate the impact of TACE-
Iodine125 treatment on the long-term survival of hepatitis Brelated HCC (HB-HCC) patients with PVTT compared with TACE alone.

\section{Materials and methods}

\section{Patients}

From 1 January 2011, to 1 June 2014, 543 consecutive patients were diagnosed with HB-HCC with PVTT in two medical centres: Lingnan Hospital and the Third Affiliated Hospital of Sun Yat-sen University. A total of 160 cases treated with TACE combined with sorafenib and 30 cases treated with sorafenib alone were excluded. Thus, 133 patients who underwent TACE-Iodine 125 and 220 patients who underwent TACE alone were assessed for eligibility in the present study. This retrospective matched cohort study was approved by the local Ethics Committee. Informed consent was obtained from all patients in this study.

Seventy patients from the pool of patients $(n=133)$ treated with TACE-Iodine 125 met the inclusion and exclusion criteria and entered the TACE-Iodine125 group. The inclusion criteria included: 1.) Positive test for the hepatitis $B$ surface antigen for 6 months; 2.) Medical imaging demonstrating type I or II PVTT, non-obstructing type III PVTT, or obstructive type III PVTT with multiple collateral vessels; 3.) Child-Pugh class A or B; and 4.) Eastern Cooperative Group performance status (ECOG) score of $0-2$. The exclusion criteria were: 1.$)$ Extrahepatic metastasis; 2.) Type IV PVTT; 3.) Hepatic encephalopathy, severe ascites, oesophageal or gastric fundal variceal bleeding or other serious medical comorbidities; 4.) Patients complicated with hepatitis A virus or hepatitis $\mathrm{C}$ virus infection or other severe diseases; 5.) Patients who underwent liver transplantation during this study; and 6.) Patients who had malignant tumours in addition to HCC.

One hundred and forty patients were selected from the pool of patients $(n=220)$ treated with TACE alone and entered the controlled group (TACE group). According to the principles of the matched cohort study, the patients in the TACE group were matched with the patients in the TACE-Iodine 125 group at a 1:2 ratio based on age (within 5 years) at TACE, gender, baseline type of PVTT, Child-Pugh class (A or B), and BCLC stage (stage $\mathrm{C}$ without extrahepatic metastasis).

HCC was diagnosed if the liver lesions (at least $1 \mathrm{~cm}$ in diameter) in a hepatitis patient showed characteristic vascular features in dynamic imaging studies: arterial hypervascularization with washout in the portal venous or the late phase [16]. The presence of PVTT was confirmed by the observation of a low-attenuation intraluminal mass expanding into the main portal vein and/or portal vein branches with three-phase dynamic computed tomography (CT) [17]. PVTT was categorized into four types according 
to the PVTT classification proposed by Shi et al. [18]: type I, PVTT in segmental portal vein; type II, PVTT affecting left/right portal vein; type III, PVTT affecting main portal vein; and type IV, PVTT affecting superior mesenteric vein.

\section{PVTT treatment strategy}

For BCLC stage C HCC patients with PVTT, TACE-sorafenib was recommended first if the main portal vein was patent or multiple collateral vessels of the obstructed portal vein were observed in the CT or magnetic resonance (MR) imaging. Sorafenib was only recommended for patients with a completely obstructed portal vein and absent collateral vessels observed in the CT or MRI imaging. TACE-Iodine 125 was recommended for patients who refused TACE-sorafenib; otherwise, TACE alone was performed for patients who refused TACE-sorafenib and TACE-Iodine125, or had a contraindication for Iodine-125 seed implantation (prothrombin time $>17$ seconds).

\section{TACE procedure}

The TACE procedure was performed as described previously [19]. Briefly, TACE was performed when the tip of the microcatheter was advanced into the hepatic segmental or specific tumour-feeding artery. An emulsion of 2-20 mL of lipiodol (Lipiodol Ultrafluido, Guerbet, Roissy, France) and 20-60 mg of doxorubicin hydrochloride were administered into the feeding vessels, and a $50 \mathrm{mg}$ solution of lobaplatin (Hainan Changan International Pharmaceutical, China) was infused into the hepatic artery. If the flow of the tumourfeeding artery was still fast after the $20 \mathrm{ml}$ upper limit was reached, we used a gelatine sponge or polyvinyl alcohol particles to embolize the vessel until we could observe a significantly sluggish flow. In patients with an arterioportal shunt (APS), an embolization with 300-1000 $\mu$ m polyvinyl alcohol particles was performed to occlude the shunt via superselective catheterization prior to infusion of the lipiodol emulsion.

\section{Iodine125 seed implantation}

Iodine 125 seed implantation into the PVTT was conducted 7 10 days after TACE when the results of the liver function tests were comparable to those obtained before TACE. The Iodine 125 seeds were provided by the Beijing ZHIBO BioMedical Technology Company, China. Each particle was $4.5 \mathrm{~mm}$ in length and $0.8 \mathrm{~mm}$ in diameter, with a radioactivity of $0.6-0.8 \mathrm{mCi}$, radioactive half-life of 60.1 days, and radiation energy of $27.4 \mathrm{KeV}$. Pre-procedural planning was conducted using a three-dimensional conformal radiation therapy treatment planning system (TPS) to determine the number of Iodine 125 seeds required, the target location for implantation, the best percutaneous puncture site and the access route. The targeted zone for implantation was the tumour thrombosis in the segmental portal vein, left/right portal vein or main portal vein. The prescription dose (PD) was set to $120 \mathrm{~Gy}$. Implantation was guided by CT, and the Iodine 125 seeds were implanted into the PVTT using $18 \mathrm{G}$ needles and the implantation gun that housed the Iodine 125 seeds in the cartridge chamber. Manual compression was applied to the percutaneous access site after the removal of the puncture needle.

\section{Follow-up and retreatment}

All patients were routinely followed up with contrast enhanced CT/MRI every 4-8 weeks after the first treatment. The laboratory tests were conducted within one week before and after the first TACE or Iodine 125 seed implantation and every 4-8 weeks during follow-up. At each visit, TACE was repeated if the following criteria were reached: 1.) CT/MRI imaging indicating viable tumour tissue at the original site or a de novo neoplasm in the hepatic parenchyma; and 2.) Good hepatic function reserve (Child-Pugh class A or B) and no contraindication for TACE.

\section{Treatment evaluation}

All adverse events (AEs) were graded in accordance with the Common Toxicity Criteria Adverse Events Version (CTCAE) 3.0. Mild subcapsular haemorrhage was defined as an asymptomatic haemorrhage with clinically confirmed observations not requiring intervention. All CT/MRI imaging evaluations were conducted by two independent experienced radiologists. The tumour response was assessed according to the modified Response Evaluation Criteria in Solid Tumours (mRECIST) [20]. The PVTT radiologic response was assessed as a complete response (CR, complete disappearance), partial response (PR, $>50 \%$ decrease in the thrombus diameter), stable disease (SD, $<50 \%$ decrease or $<25 \%$ increase in the thrombus diameter or cavernous transformation), and progressive disease (PD, $>25 \%$ increase in the thrombus diameter or newly developed PVTT), based on the criteria described by Yoon et al. [21]. The disease control rate was defined as the percentage of CR, PR, or SD patients.

\section{Power analysis}

Sample sizes of 70 in the TACE-Iodine 125 group and 140 in the TACE group ensured at least $80 \%$ power at a $5 \%$ significance level to detect significant differences between the groups when the hazard ratio of treatment over control was 0.58 or smaller for the log-rank test. For the Cox regression analysis, these sample sizes ensured at least $80 \%$ power at a $5 \%$ significance to detect significant binary predictors if the 
adjusted hazard ratio was 0.55 and the r-square between this factor and other factors in the model was 0.3 or less.

\section{Statistical analysis}

Quantitative data were reported as the mean $\pm \mathrm{SD}$ and compared between two groups using Student's $t$-test. Categorical data were compared using the $\chi 2$ test. Survival was analysed using a Kaplan-Meier curve and log-rank test. Overall survival (OS) was defined as the period from the time of the first TACE procedure until death or the last follow-up. The Cox proportional hazard model was used for univariate and multivariate analyses to determine prognostic factors and the adjusted effect of the TACE-Iodine125 group over the control. Factors significant at the 0.20 level in the univariate analysis were chosen as candidates for the multivariate analysis. The SPSS13.0 software (SPSS, Chicago, IL, USA) for statistical analysis was used for all analyses. Differences were deemed significant when $p \leq 0.05$.

\section{Results}

\section{Baseline information and clinical outcome}

The baseline demographic characteristics of patients were shown in Table 1. There was no significant difference in the baseline demographic characteristics between the TACEIodine 125 and TACE groups $(p>0.05)$. The mean number of TACE procedures performed over the study period was $3.3 \pm 1.7(1-8)$ and $3.1 \pm 1.7(1-8)$ for the TACE-Iodine 125 and TACE groups, respectively. A radiologic response was noted in 33 (1 CR, $16 \mathrm{PR}$ and $16 \mathrm{SD}, 47.1 \%$ ) cases in the TACE-Iodine125 group (Fig. 1a-d) and 45 (0 CR, 20 PR and $25 \mathrm{SD}, 32 \%$ ) cases in the TACE group, representing a significant difference $(p=0.034)$. A PVTT radiologic response was observed in 53 ( 0 CR, 38 PR and $15 \mathrm{SD}, 76 \%)$ cases in the TACE-Iodine 125 group and $69(0 \mathrm{CR}, 30 \mathrm{PR}$ and $39 \mathrm{SD}$, $49 \%$ ) cases in the TACE group, representing a significant different between the two groups $(p<0.001)$. The numbers of TACE procedures in the PVTT responders and PVTT non-responders in the TACE-Iodine125 group were $3.3 \pm 1.6$ $(1-8)$ and $3.5 \pm 2.0(2-7)$, respectively; there was no significant difference between the groups ( $p=0.719)$.

\section{Complications}

The AEs of the two groups were detailed in Table 2. Grade 1 and 2 AEs were relatively mild and were alleviated spontaneously or after conservative management for only 5-7 days post-procedure. Serious AEs (SAEs) such as biloma were successfully managed by percutaneous puncture and draining. No other SAEs were observed in either of the groups, and there was no procedure-related mortality.

\section{Survival}

The mean and median follow-up times were $9.3 \pm 5.3$ months and $8.0(3.6-43.0)$ months in the TACE-Iodine125 group and $8.4 \pm 5.9$ months and $6.0(3.5-37.0)$ months in the TACE group, respectively. Altogether, 138 patients died during follow-up (28 in the TACE-Iodine125 group and 110 in the TACE group). The median OS was 11 months in the TACE-Iodine125 group and 7.5 months in the TACE group. The 6-, 12-, 24-, and 36-month survival rates were $85 \%, 50 \%, 14.5 \%$, and $14.5 \%$ in the TACE-Iodine 125 group and $55 \%, 25 \%, 9 \%$, and $5 \%$ in the TACE group, respectively. The survival curves demonstrated better outcomes in the TACE-Iodine125 group compared to the TACE group (Fig. 2, $p<0.001$ ).

The survival times of type I and II PVTT patients were significantly longer than type III PVTT patients (Fig. 3a, $p<0.001)$. Further analysis showed that better outcomes were obtained for both type I + II and type III PVTT patients in the TACE-Iodine 125 group compared to the TACE group (Fig. $3 \mathrm{~b}$ and $\mathrm{c}, p=0.006$ and $p<0.001$, respectively).

The OS of patients with a PVTT radiologic response was better than the OS of patients who had no PVTT response (Fig. $4 \mathrm{a}, p<0.001$ ). For patients with no PVTT response, the survival curves showed that there were no significant differences between the groups (Fig. 4b, $p=0.353$ ).

\section{Univariate and multivariate analyses}

Univariate COX model analysis was applied to the factors involved in the preliminary screening. After screening, nine factors were selected as candidates for multivariate analysis (Table 3): treatment modality, PVTT type, PVTT response, ECOG, level of HBV DNA, number of tumours, tumour size, APS, and total bilirubin (TBIL). The multivariate Cox proportional hazard model showed that type III PVTT [hazard Ratio $(\mathrm{HR})=0.310 ; 95 \%$ confidence interval $(\mathrm{CI}): 0.212-0.453$; $p<0.001]$, and APS $(\mathrm{HR}=2.137 ; 95 \% \mathrm{CI}: 1.441-3.170$; $p<0.001$ ) were associated with a poor prognosis. After adjustment for these risk factors, the PVTT response $(\mathrm{HR}=0.503$; $95 \%$ CI: $0.338-0.748 ; p=0.001)$ and the treatment modality of TACE-Iodine125 (HR $=0.359 ; 95 \%$ CI: 0.22-0.571; $p<0.001)$ were associated with a favourable prognosis (Table 3) compared to TACE alone.

\section{Discussion}

Current treatment recommendations for $\mathrm{HCC}$ provide very limited data on PVTT management. Despite the controversy, TACE remains the first-line treatment for BCLC stage C HCC patients in China. In the present study, all HCC participants successfully received TACE treatment without serious complications (i.e., TACE-related liver failure). Notably, the 
Table 1 Baseline characteristics of HB-HCC patients in two groups

\begin{tabular}{llll}
\hline & $\begin{array}{l}\text { TACE-Iodine125 } \\
(n=70)\end{array}$ & $\begin{array}{l}\text { TACE } \\
(n=140)\end{array}$ & $p$ value \\
\hline Age, year & $51.1 \pm 11.1(28 \sim 80)$ & $51.6 \pm 10.8(22 \sim 81)$ & 0.748 \\
Sex (M/F) & $63 / 7$ & $127 / 13$ & 0.868 \\
Type of PVTT (I+II/III) & $41 / 29$ & $82 / 58$ & 1.000 \\
APS (+/-) & $20 / 50$ & $39 / 101$ & 0.914 \\
Child-Pugh class (A/B) & & & 0.558 \\
A (A5, A6) & $31(15,16)$ & $68(35,33)$ & \\
B (B7, B8, B9) & $39(27,9,3)$ & $72(54,16,2)$ & \\
ECOG (0/1+2) & $41 / 29$ & $79 / 61$ & 0.767 \\
Tumor size (cm), $<7 / \geq 7$ & $39 / 31$ & $83 / 57$ & 0.621 \\
Number of Tumor, $<3 / \geq 3$ & $30 / 40$ & $70 / 70$ & 0.329 \\
AFP level (ng/ml), $<400 / \geq 400$ & $31 / 39$ & $59 / 81$ & 0.767 \\
HBV DNA (IU/ml), $<104 / \geq 104$ & $39 / 31$ & $64 / 76$ & 0.172 \\
ALT, U/L & $71.5 \pm 32.6$ & $71.2 \pm 39.3$ & 0.951 \\
GGT, U/L & $168.7 \pm 54.0$ & $157.4 \pm 52.9$ & 0.149 \\
Albumin, g/L & $36.1 \pm 3.3$ & $35.7 \pm 2.9$ & 0.353 \\
TBIL, umol/L & $15.9 \pm 7.4$ & $16.3 \pm 7.6$ & 0.683 \\
Creatinine, umol/L & $67.9 \pm 14.7$ & $65.3 \pm 16.9$ & 0.261 \\
Platelet count, 109/L & $118.6 \pm 67.4$ & $126.8 \pm 55.1$ & 0.345 \\
Hemoglobin, g/L & $124.0 \pm 12.2$ & $121.6 \pm 14.8$ & 0.235 \\
White blood cell, 109/L & $4.70 \pm 1.05$ & $4.61 \pm 0.90$ & 0.529 \\
\hline
\end{tabular}

following steps were crucial for the performance of TACE for HCC-PVTT patients in this study. First, the careful selection of qualified patients was crucial for the success of TACE. Only patients with CHILD-PUGH A/B and ECOG 0-2 whose blood supply in the main portal vein was normal or incompletely disrupted or had imaging-confirmed portal-collateral circulation were indicated for TACE in our study. Second, all TACE procedures were carefully performed with a superselective catheterization technique. For patients with PVTT, some studies have also shown that using less aggressive embolization after superselective catheterization is safe, with no increase in morbidity or mortality $[22,23]$.
Fig. 1 Male, 57 years old, BCLC C stage HCC. (a) Contrast enhanced CT cross sectional image demonstrating a $\mathrm{HCC}$ lesion in segment 6 and PVTT reaching the main portal vein (white arrow). (b) Angiogram from the celiac trunk demonstrating the plexiform feeding artery of PVTT and APS (black arrow). (c) CT-guided Iodine 125 seed implantation in PVTT. (d) CT images acquired 28 months after the initial treatment demonstrate no viable tumour and the disappearance of PVTT and the patent portal vein a

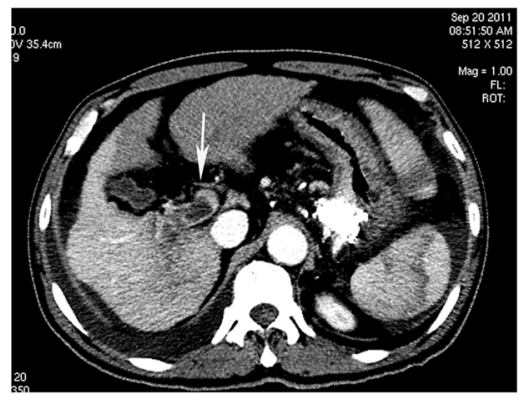

C

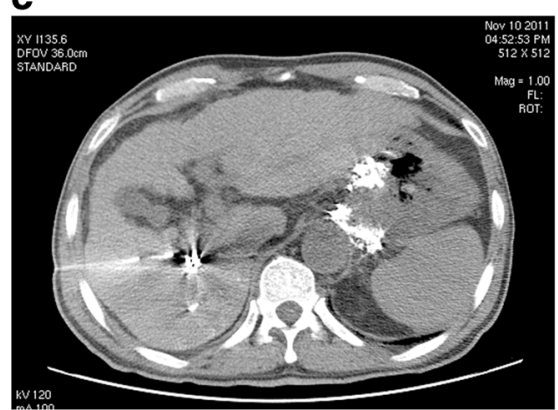

b

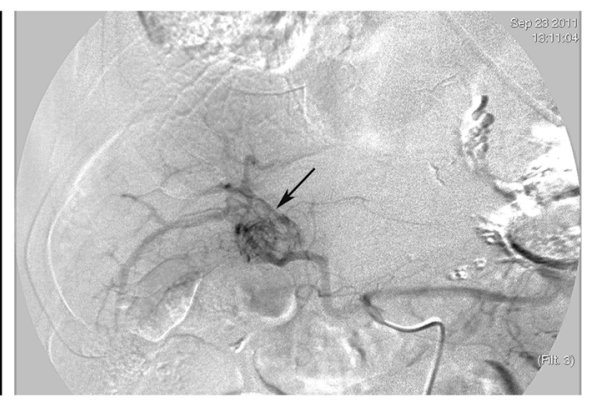

d

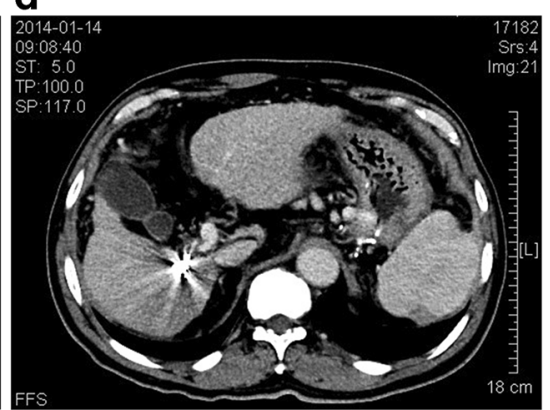


Table 2 The adverse effects of two groups in accordance with the Common Terminology Criteria for Adverse Events version 3.0

\begin{tabular}{|c|c|c|c|c|}
\hline Groups & All Grade & Grade $1-2$ & Grade3 or Higher levels & $p$ value \\
\hline Fever & & & & 0.419 \\
\hline TACE-I125 & $56(80.0 \%)$ & $56(80.0 \%)$ & 0 & \\
\hline TACE & $105(75.0 \%)$ & $105(75.0 \%)$ & 0 & \\
\hline Abdominal pain & & & & 0.099 \\
\hline TACE-I125 & $63(90.0 \%)$ & $63(90.0 \%)$ & 0 & \\
\hline TACE & $98(70.0 \%)$ & $98(70.0 \%)$ & 0 & \\
\hline Nausea and/or vomiting & & & & 0.518 \\
\hline TACE-I125 & $41(58.6 \%)$ & $41(58.6 \%)$ & 0 & \\
\hline TACE & $77(55.0 \%)$ & $77(55.0 \%)$ & 0 & \\
\hline Subcapsular hemorrhage of liver & & & & 0.008 \\
\hline TACE-I125 & $3(4.3 \%)$ & $3(4.3 \%)$ & 0 & \\
\hline TACE & 0 & 0 & 0 & \\
\hline Biloma & & & & 0.477 \\
\hline TACE-I125 & $4(5.7 \%)$ & - & $4(5.7 \%)$ & \\
\hline TACE & $5(3.6 \%)$ & - & $5(3.6 \%)$ & \\
\hline ALT, AST and/or TBIL Increased & & & & 1.000 \\
\hline TACE-I125 & $70(100 \%)$ & $70(100 \%)$ & 0 & \\
\hline TACE & $140(100 \%)$ & $140(100 \%)$ & 0 & \\
\hline
\end{tabular}

Although TACE has been proven to be an effective and safe basic therapeutic method for intrahepatic primary HCC lesions, combined treatment with TACE and novel drugs or other therapies might be a better alternative strategy for HCC with PVTT. TACE combined with sorafenib was considered to be an effective combination treatment for HCC-PVTT [24] and was first recommended by the attending physician in our institute if the main portal vein was patent or multiple collateral vessels from the obstructed portal vein were observed in the CT or MRI imaging [19]. Nevertheless, sorafenib does not represent a cost-effective drug in this indication in some developing countries [25]. Additionally, some patients are afraid

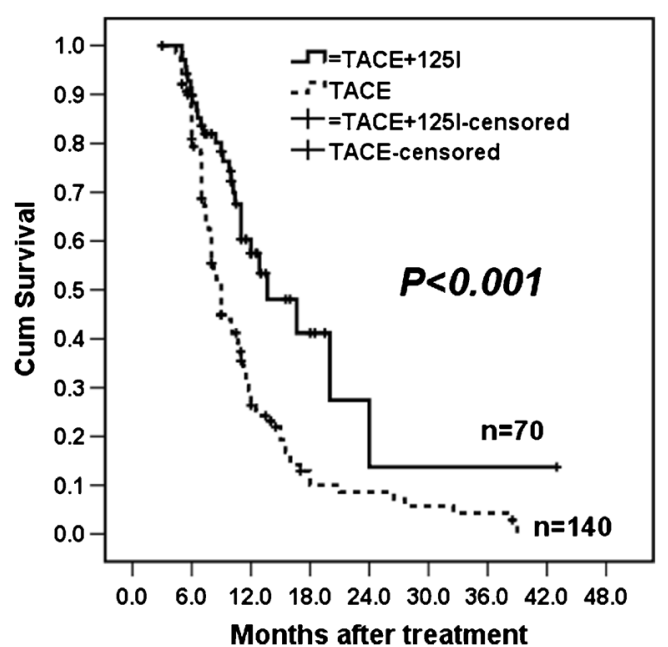

Fig. 2 Kaplan-Meier estimates of OS between the TACE-Iodine125 and TACE groups of a deterioration of their quality of life owing to drug-induced side effects, and consequently refuse to take sorafenib. In this study, although we recommended that all HCC-PVTT patients take sorafenib as a standard treatment, these patients refused for the above reasons and consented to TACE alone or TACE plus Iodine 125 seed implantation.

There is increasing evidence that Yttrium-90 radioactive microsphere hepatic artery embolization (TARE) can be delivered safely and effectively in HCC-PVTT patients, leading to a median OS of approximately 10 months following the procedure [26]. TARE might have an advantage over TACE in Child-Pugh A patients with very large tumours where TACE does not provide a very effective treatment [27]. Similar to Yttrium-90, Iodine131 is another $\beta$-emitter with a physical half-life of 8.05 days that can be used as a radionuclide therapy for HCC and PVTT. Because Iodine131-lipiodol is a noparticle embolization at the end of the procedure, PVTT is not considered to be a relative or absolute contraindication for Iodine131lipiodol. Although most studies have failed to demonstrate any survival benefits, Iodine131-lipiodol seems to be much better tolerated than chemoembolization, both in terms of clinically expressed side effects and arteriographic findings [28]. LintiaGaultier et al [29] reported a median survival of 32 weeks in fifty patients ( $86 \%$ of them complicated with PVTT) treated with Iodine131-lipiodol compared with 8 weeks in the untreated group. Raoul et al. [30] reported a similar result of a median survival of 6 months in the Iodine131-lipiodol group compared with 1.5 months in the control group for Child-Pugh A and B patients with PVTT. However, the treatment tolerance and effectiveness of radioembolization or Iodine131-lipiodol may be 

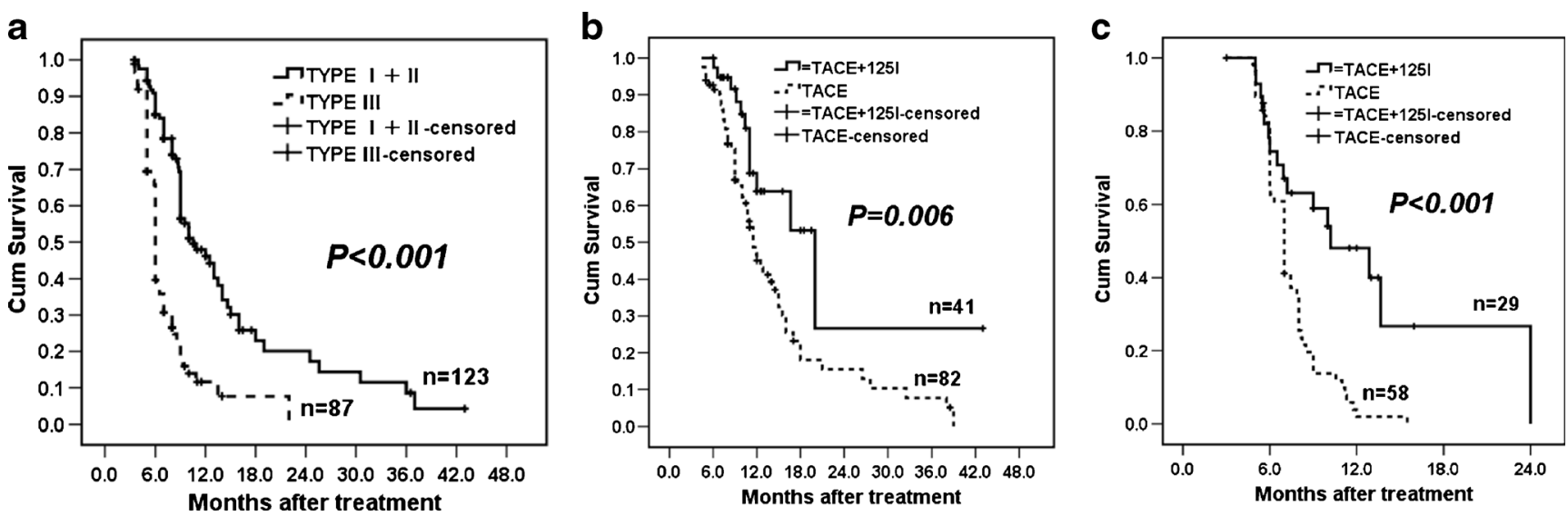

Fig. 3 Kaplan-Meier estimates of (a) OS between type I + II and III PVTT patients, (b) OS between the TACE-Iodine125 and TACE groups in type I + II PVTT patients, and (c) OS between the TACE-Iodine125 and TACE groups in type III PVTT patients

affected when it is used for cirrhotic patients or patients with APS or arterio-venous shunts (AVS) [31]. Three-dimensional conformal radiation therapy (3-DCRT) is another novel radiotherapy for PVTT [13]. Tang et al. [32] reported that combined TACE and 3-DCRT resulted in a median survival time of 12.3 months and survival rates of $51.6 \%, 28.4 \%$, and $19.9 \%$ at 1,2 , and 3 years, respectively, among 185 HCCPVTT cases. However, no prospective study has compared Iodine-131-lipiodol, TARE, 3-DCRT and Iodine-125 seed implantation in HCC patients with PVTT.

Yang et al. [33] adopted a strategy of TACE combined with intravascular implantation of Iodine 125 seed strands into the portal vein for the treatment of 43 HCC-PVTT patients and reported that the average and median survival times were $221.7 \pm 16.3$ days and $210.0 \pm 17.5$ days, respectively. However, the potential problem associated with the intravascular implantation of radioactive seed strands is that insufficient irradiation of PVTT may occur due to the uneven distribution of the Iodine 125 seed strand radioactive sources inside the PVTT. Based on the pre-procedural TPS planning and CT scan surveillance during the procedure, percutaneous transhepatic puncture of the PVTT and implantation of
Iodine125 seeds inside and around the PVTT could allow an even and quantifiable distribution of the radioactive source.

Our results showed that the OS of patients who had a radiologic response to PVTT was more favourable than patients who had no response to PVTT. There were no significant differences in the survival of patients with no response to PVTT between the TACE-I125 and TACE groups. Our results indicated that the separate treatment of PVTT was very important for prolonging the survival time of patients with $\mathrm{HCC}$ and PVTT. We speculate that the effective treatment of PVTT can partially or completely reopen the portal venous system, which can in turn maintain the oxygen supply to the liver, induce a good state of liver function, and effectively reduce the incidence of portal hypertension-related complications. Additionally, effective PVTT treatment can also prevent or delay intrahepatic metastasis and tumour recurrence.

APS was considered to be another independent predictor of poor prognosis and a typical complication of PVTT $[12,34]$. Although the exact reason was unknown, some studies found that APS could be decreased or cured with radiotherapy [31]. However, future studies are required to prove that Iodine 125 implantation in the PVTT can lead to a good APS response.
Fig. 4 Kaplan-Meier estimates of (a) OS between PVTT responders and non-responders and (b) OS between PVTT non-responders in the TACE-Iodine 125 and TACE groups a

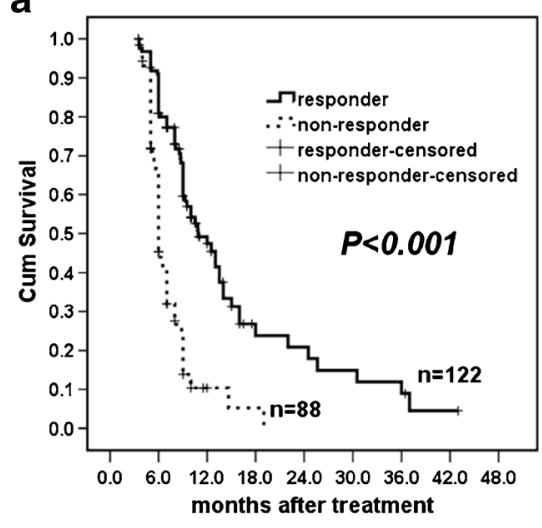

b

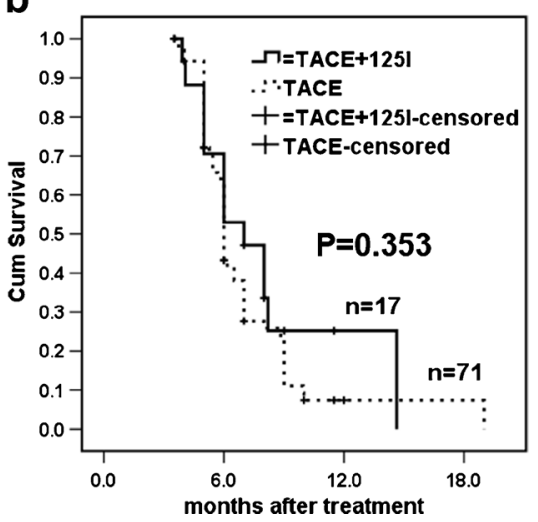


Table 3 Univariate and multivariate analyses of factors that may affect overall survival of HB-HCC patients with PVTT

\begin{tabular}{|c|c|c|c|c|c|c|}
\hline \multirow[t]{2}{*}{ Risk factor } & \multicolumn{3}{|c|}{ Univariate } & \multicolumn{3}{|c|}{ Multivariate } \\
\hline & HR & $95 \% \mathrm{CI}$ & $p$ value & $\mathrm{HR}$ & $95 \% \mathrm{CI}$ & $p$ value \\
\hline Gender (M/F) & 0.773 & $0.463 \sim 1.290$ & 0.324 & & & \\
\hline Age (Y) & 1.003 & $0.987 \sim 1.019$ & 0.725 & & & \\
\hline Type of PVTT (I + II/III) & 0.311 & $0.218 \sim 0.444$ & 0.000 & 0.310 & $0.212 \sim 0.453$ & 0.000 \\
\hline Response to PVTT & 0.312 & $0.218 \sim 0.445$ & 0.000 & 0.503 & $0.338 \sim 0.748$ & 0.001 \\
\hline HBV DNA(IU/ml, $<104 / \geq 104$ & 0.738 & $0.526 \sim 1.036$ & 0.079 & 0.965 & $0.680 \sim 1.370$ & 0.841 \\
\hline $\operatorname{APS}(+/-)$ & 1.979 & $1.353 \sim 2.894$ & 0.000 & 2.137 & $1.441 \sim 3.170$ & 0.000 \\
\hline Child-Pugh class, A/B & 0.892 & $0.637 \sim 1.249$ & 0.506 & & & \\
\hline ECOG, $0 / 1+2$ & 0.480 & $0.336 \sim 0.685$ & 0.000 & 0.810 & $0.537 \sim 1.220$ & 0.313 \\
\hline Number of tumor, $<3 / \geq 3$ & 0.721 & $0.515 \sim 1.010$ & 0.057 & 0.763 & $0.510 \sim 1.143$ & 0.190 \\
\hline Tumor size $(\mathrm{cm}),<7 / \geq 7$ & 0.796 & $0.569 \sim 1.114$ & 0.184 & 0.817 & $0.577 \sim 1.156$ & 0.254 \\
\hline $\operatorname{WBC}(109 / \mathrm{L}),<4 / \geq 4$ & 1.078 & $0.754 \sim 1.541$ & 0.680 & & & \\
\hline Hemoglobin $(\mathrm{g} / \mathrm{L}),<100 / \geq 100$ & 1.120 & $0.688 \sim 1.823$ & 0.649 & & & \\
\hline Platelet count $(109 / \mathrm{L}),<100 / \geq 100$ & 1.016 & $0.719 \sim 1.436$ & 0.929 & & & \\
\hline $\operatorname{TBIL}(\mu \mathrm{mol} / \mathrm{L}),<20 / \geq 20$ & 0.791 & $0.555 \sim 1.126$ & 0.194 & 0.943 & $0.655 \sim 1.356$ & 0.750 \\
\hline Albumin $(\mathrm{g} / \mathrm{L},<35 / \geq 35$ & 0.836 & $0.595 \sim 1.173$ & 0.300 & & & \\
\hline $\operatorname{ALT}(\mathrm{U} / \mathrm{L}),<35 / \geq 35$ & 1.152 & $0.819 \sim 1.622$ & 0.417 & & & \\
\hline GGT $(\mathrm{U} / \mathrm{L}),<60 / \geq 60$ & 1.163 & $0.827 \sim 1.636$ & 0.385 & & & \\
\hline $\operatorname{AFP}(\mathrm{ng} / \mathrm{mL}),<400 / \geq 400$ & 0.842 & $0.599 \sim 1.184$ & 0.323 & & & \\
\hline Treatment modality & 0.405 & $0.267 \sim 0.615$ & 0.000 & 0.405 & $0.267 \sim 0.615$ & 0.000 \\
\hline
\end{tabular}

Some studies showed that Child-Pugh B patients had significantly poorer survival compared to Child-Pugh A patients [35]. This present study did not demonstrate a favourable survival benefit in Child-Pugh A compared with Child-Pugh B patients, which may be related to the fact that the $81(73 \%)$ Child-Pugh B patients in this study had a Child-Pugh numeric score of 7 (B7). A previous study demonstrated that Child-Pugh B7 patients had a larger survival rate than Child-Pugh B8 and B9 patients [36]. Piscaglia et al. [36] reported that Child-Pugh B7 HCC patients achieved an acceptable median survival of 22.0 months (range 0-60.0) after TACE. That result was comparable to the results included in Llovet's meta-analysis [37], which was largely based on Child-Pugh A patients. In another retrospective study by Federico et al. [38], the OS in Child-Pugh A patients was not significantly different compared to Child-Pugh B7 patients. Future trials should validate this observation.

The main limitation of retrospective studies is selection bias. To reduce bias caused by using the TACE group as the control, the factors most likely to affect the survival outcome were used as matching factors to select a matching cohort of patients. However, the retrospective nature of the study dictated that selection bias was unavoidable. A randomized clinical trial is required to validate the findings from this study. Furthermore, only patients with HB-HCC were studied here. Thus, the results from this study may not be applicable to other HCC populations.

In conclusion, combined treatment with TACE and Iodine 125 seed implantation achieved a promising OS in selected unresectable HB-HCC patients with PVTT. Therefore,
TACE combined with Iodine 125 seed implantation might be an alternative treatment for these patients. A randomized clinical trial is warranted to validate the findings of this study.

Acknowledgments The scientific guarantor of this publication is Hong Shan, PHD., the head of the Department of Radiology, the Third Affiliated Hospital, Sun Yat-Sen University, China. The authors of this manuscript declare no relationships with any companies, whose products or services may be related to the subject matter of the article. This study has received funding by the National Natural Science Foundation of China (81172193 and 81430041), and the Scientific Research Foundation for the Returned Overseas Chinese Scholars, State Education Ministry. Prof Zhao-Xing Pan and Li Ling kindly provided statistical advice for this manuscript. Institutional Review Board approval was obtained. Written informed consent was obtained from all subjects (patients) in this study. Methodology: retrospective, case-control study, multicenter study.

Open Access This article is distributed under the terms of the Creative Commons Attribution-NonCommercial 4.0 International License (http:// creativecommons.org/licenses/by-nc/4.0/), which permits any noncommercial use, distribution, and reproduction in any medium, provided you give appropriate credit to the original author(s) and the source, provide a link to the Creative Commons license, and indicate if changes were made.

\section{References}

1. Jemal A, Bray F, Center MM, Ferlay J, Ward E, Forman D (2011) Global cancer statistics. CA Cancer J Clin 61:69-90

2. Li W, Chen G, Yu X, Shi Y, Peng M, Wei J (2013) Accumulation of the mutations in basal core promoter of hepatitis B virus 
subgenotype $\mathrm{C} 1$ increase the risk of hepatocellular carcinoma in Southern China. Int J Clin Exp Pathol 6:1076-1085

3. Llovet JM, Bustamante J, Castells A et al (1999) Natural history of untreated nonsurgical hepatocellular carcinoma: rationale for the design and evaluation of therapeutic trials. Hepatology 29:62-67

4. Cabibbo $\mathrm{G}$, Enea $\mathrm{M}$, Attanasio $\mathrm{M}$, Bruix J, Craxì A, Cammà $\mathrm{C}$ (2010) A meta-analysis of survival rates of untreated patients in randomized clinical trials of hepatocellular carcinoma. Hepatology 51:1274-1283

5. Bruix J, Sherman M (2011) American association for the study of liver D. Management of hepatocellular carcinoma: an update. Hepatology 53:1020-1022

6. Llovet JM, Di Bisceglie AM, Bruix J et al (2008) Design and endpoints of clinical trials in hepatocellular carcinoma. J Natl Cancer Inst 100:698-711

7. Cheng AL, Kang YK, Chen Z et al (2009) Efficacy and safety of sorafenib in patients in the Asia-Pacific region with advanced hepatocellular carcinoma: a phase III randomised, double-blind, placebo-controlled trial. Lancet Oncol 10:25-34

8. Llovet JM, Ricci S, Mazzaferro V et al (2008) Sorafenib in advanced hepatocellular carcinoma. N Engl J Med 359:378-390

9. Omata M, Lesmana LA, Tateishi R et al (2010) Asian pacific association for the study of the liver consensus recommendations on hepatocellular carcinoma. Hepatol Int 4:439-474

10. Luo J, Guo RP, Lai EC et al (2011) Transarterial chemoembolization for unresectable hepatocellular carcinoma with portal vein tumor thrombosis: a prospective comparative study. Ann Surg Oncol 18: 413-420

11. Georgiades CS, Hong K, D'Angelo M, Geschwind JF (2005) Safety and efficacy of transarterial chemoembolization in patients with unresectable hepatocellular carcinoma and portal vein thrombosis. J Vasc Interv Radiol 16:1653-1659

12. Huang MS, Lin Q, Jiang ZB et al (2004) Comparison of long term effects between intra-arterially delivered ethanol and Gelfoam for the treatment of severe arterioportal shunt in patients with hepatocellular carcinoma. World J Gastroenterol 10:825-829

13. Kim DY, Park W, Lim Do H et al (2005) Three-dimensional conformal radiotherapy for portal vein thrombosis of hepatocellular carcinoma. Cancer 103:2419-2426

14. Zhang FJ, Li CX, Jiao DC et al (2008) CT guided 125iodine seed implantation for portal vein tumor thrombus in primary hepatocellular carcinoma. Chin Med J 121:2410-2414

15. Denecke T, Stelter L, Schnapauff D et al (2015) CT-guided interstitial brachytherapy of hepatocellular carcinoma before liver transplantation: an equivalent alternative to transarterial chemoembolization? Eur Radiol 25:2608-2616

16. Bruix J, Sherman M (2005) Practice guidelines committee, American association for the study of liver diseases. Management of hepatocellular carcinoma. Hepatology 42:1208-1236

17. Chung JW, Park JH, Han JK, Choi BI, Han MC (1995) Hepatocellular carcinoma and portal vein invasion: results of treatment with transcatheter oily chemoembolization. AJR Am J Roentgenol 165:315-321

18. Shi J, Lai EC, Li N et al (2010) Surgical treatment of hepatocellular carcinoma with portal vein tumor thrombus. Ann Surg Oncol 17: 2073-2080

19. Zhu KS, Chen JW, Lai LS et al (2014) Hepatocellular carcinoma with portal vein tumor thrombus: treatment with transarterial chemoembolization combined with sorafenib-A retrospective controlled study. Radiology 272:284-293

20. Lencioni R, Llovet JM (2010) Modified RECIST (mRECIST) assessment for hepatocellular carcinoma. Semin Liver Dis 30:52-60
21. Yoon SM, Lim YS, Won HJ et al (2011) Radiotherapy plus transarterial chemoembolization for hepatocellular carcinoma invading the portal vein: long-term patient outcomes. Int J Radiat Oncol Biol Phys 82:2004-2011

22. Zhang YF, Guo RP, Zou RH (2015) Efficacy and safety of preoperative chemoembolization for resectable hepatocellular carcinoma with portal vein invasion: a prospective comparative study. Eur Radiol

23. Kothary N, Weintraub JL, Susman J, Rundback JH (2007) Transarterial chemoembolization for primary hepatocellular carcinoma in patients at high risk. J Vasc Interv Radiol 18:1517-1526

24. Abou-Alfa GK (2011) TACE and sorafenib: a good marriage? J Clin Oncol 29:3949-3952

25. Duerden $M$ (2010) From a cancer drug fund to value based pricing of drugs. BMJ 341:c4388

26. Sangro B, Carpanese L, Cianni R et al (2011) Survival after 90Y resin microsphere radioembolization of hepatocellular carcinoma across BCLC stages: a European evaluation. Hepatology 54:868878

27. Carr BI, Kondragunta V, Buch SC, Branch RA (2010) Therapeutic equivalence in survival for hepatic arterial chemoembolization and yttrium 90 microsphere treatments in unresectable hepatocellular carcinoma: a two-cohort study. Cancer 116:1305-1314

28. Raoul JL, Guyader D, Bretagne JF et al (1997) Prospective randomized trial of chemoembolization versus intra-arterial injection of 131I-labeled-iodized oil in the treatment of hepatocellular carcinoma. Hepatology 26:1156-1161

29. Lintia-Gaultier A, Perret C, Ansquer C, Eugène T, Kraeber-Bodére F, Frampas E (2013) Intra-arterial injection of 131I-labeled lipiodol for advanced hepatocellular carcinoma: a 7 years' experience. Nucl Med Commun 34:674-681

30. Raoul JL, Guyader D, Bretagne JF et al (1994) Randomized controlled trial for hepatocellular carcinoma with portal vein thrombosis: intra-arterial iodine-131-iodized oil versus medical support. J Nucl Med 35:1782-1787

31. Sangro B, Inarrairaegui M, Bilbao JI (2012) Radioembolization for hepatocellular carcinoma. J Hepatol 56:464-473

32. Tang QH, Li AJ, Yang GM et al (2013) Surgical resection versus conformal radiotherapy combined with TACE for resectable hepatocellular carcinoma with portal vein tumor thrombus: a comparative study. World J Surg 37:1362-1370

33. Yang M, Fang Z, Yan Z et al (2014) Transarterial chemoembolisation (TACE) combined with endovascular implantation of an iodine-125 seed strand for the treatment of hepatocellular carcinoma with portal vein tumour thrombosis versus TACE alone: a two-arm, randomised clinical trial. J Cancer Res Clin Oncol 140:211-219

34. Hsu HC, Chen TY, Chiu KW et al (2007) Three-dimensional conformal radiotherapy for the treatment of arteriovenous shunting in patients with hepatocellular carcinoma. Br J Radiol 80:38-42

35. Pinter M, Hucke F, Graziadei I et al (2012) Advanced-stage hepatocellular carcinoma: transarterial chemoembolization versus Sorafenib. Radiology 263:590-599

36. Piscaglia A, Terzi E, Cucchetti A et al (2013) Treatment of hepatocellular carcinoma in Child-Pugh B patients. Dig Liver Dis 45:852858

37. Llovet JM, Real MI, Montana X et al (2002) Arterial embolisation or chemoembolisation versus symptomatic treatment in patients with unresectable hepatocellular carcinoma: a randomised controlled trial. Lancet 359:1734-1739

38. Federico A, Orditura M, Cotticelli G et al (2015) Safety and efficacy of sorafenib in patients with advanced hepatocellular carcinoma and Child-Pugh A or B cirrhosis. Oncol Lett 9:1628-1632 